\title{
The tilt illusion as a function of the relative and absolute lengths of test and inducing lines
}

\author{
PETER WENDEROTH, TONY O'CONNOR, and MICHAEL JOHNSON \\ University of Sydney, Sydney, New South Wales, Australia
}

\begin{abstract}
The explanation of the tilt illusion that attributes the effect to lateral inhibitory interactions between cortical orientation-selective neurons suggests that illusion magnitude is dependent in part upon the lengths of the inducing (I) and test (T) arms of an angle. The model predicts (1) larger illusions for I length $>\mathrm{T}$ than for $\mathrm{T}>\mathrm{I}$; (2) that when $\mathrm{I}=\mathrm{T}$, peak illusion magnitude will occur for smaller angles between $I$ and $T$ when these lines are long than when they are short; and (3) that when $\mathrm{I}=\mathrm{T}$, smaller peak illusions for long lines than for short lines. Two experiments were conducted to test these predictions, and the results were consistent with all but the last of them. It is suggested that the aspect of the model that attributes perceived line orientation to the most active neurons stimulated by a line (the mode of the response function) requires reexamination.
\end{abstract}

When two lines abut to form an acute angle, they repel each other in the orientation domain, an effect known as the tilt illusion. Carpenter and Blakemore (1973) proposed that each line maximally excites orientation-selective neurons in the visual cortex which are tuned to that line's orientation. Cells tuned to similar orientations are also excited, but less so, and cells tuned to slightly more remote orientations, which are not excited by the stimulus line, are more or less strongly inhibited by the activity of their excited neighbors. The result is that each line presented alone would induce a distribution of cortical activity resemblng a "Mexican hat," with a peak of excitation surrounded by inhibitory troughs. Presenting both lines together causes these cortical processes to interact: when the two lines form a small acute angle, the peak of excitation generated by one line coincides with the inhibitory trough induced by the other. If excitation and inhibition sum linearly, "the most active neurons for the two lines presented together are not the same as they were when they were presented singly: the peaks are shifted apart" (Carpenter \& Blakemore, 1973, p. 288). The model accounts for the tilt illusion on the assumption that the perceived orientation of a line is determined by the orientation-tuning of the most active neurons.

The basic features of the Carpenter and Blakemore model are summarized in Figure $1 \mathrm{~A}$.

A pair of stimulus lines, $A$ and $B$, are located in the orientation domain such that they form an acute angle of, say, $16^{\circ}$ (see inset in Figure 1A). Line A, when presented alone, induces the dashed distribution of cortical activity

\footnotetext{
This research was supported by the Australian Research Grants Scheme, Grant A28515620 I to the first author. We thank John Holden for assistance with computing facilities, G. Stuart for producing the functions in Figures 1 and 2, and Bogdan Dreher for discussion of the relationship between single-cell and population-tuning curves.

P. Wenderoth and Tony O'Connor are in the Department of Psychology and M. Johnson is in the Department of Pure Mathematics at the University of Sydney, Sydney, N.S.W., Australia 2006.
}

with peak excitation at $\mathrm{A}$. The excitatory distribution is surrounded by inhibitory flanks, and these, in turn, are bordered by disinhibitory flanks (cf. O'Toole \& Wenderoth, 1977). Similarly, line B alone induces the dashed distribution of activity with its excitatory peak at B. When the two lines are presented together as an acute angle, simple linear summation of the two dashed distributions produces the solid function, which has peaks at $A^{\prime}$ and $B^{\prime}, 18^{\circ}$ apart, resulting in an illusory angle expansion of $2^{\circ}$.

Conventionally, the tilt illusion is measured by judging the orientation of just one line (A, the test line). According to Wallace (1975), any parameter of the other line (B, the inducing line) that increases its inhibitory output would increase the magnitude of the peak shift, from A to $\mathrm{A}^{\prime}$. According to Wallace, the shift in the excitatory peak, and hence the magnitude of the illusion, "is due to the differences in slope between the excitation distribution near its peak and that of the inhibition distribution at this point. As the contrast [of the inducing line] is increased the inhibition slope is increased and the peak shift is increased" (Wallace, 1975, p. 965).

Using the Zöllner illusion, in which the test line (T) is intersected by many inducing (I) lines at a nearby orientation, Wallace demonstrated that illusion magnitude was a linear function of the log contrast of the inducing lines. He concluded that the model represented in Figure 1 accounted for his results, and that the illusion probably arises due to interactions between simple cortical cells, because only these, in the cat, show a proportional relationship between frequency of firing and log contrast.

Parker (1974), using the tilt illusion, extended these findings by measuring the change in perceived orientation of line A (Figure 1, inset) as (1) the luminance (and hence contrast) of $B$ was increased relative to the luminance of $A,(2)$ the luminance of $A$ was increased relative to that of $B$, and (3) the luminance of both $A$ and $B$ were jointly increased. He found, as he predicted, that 
A.
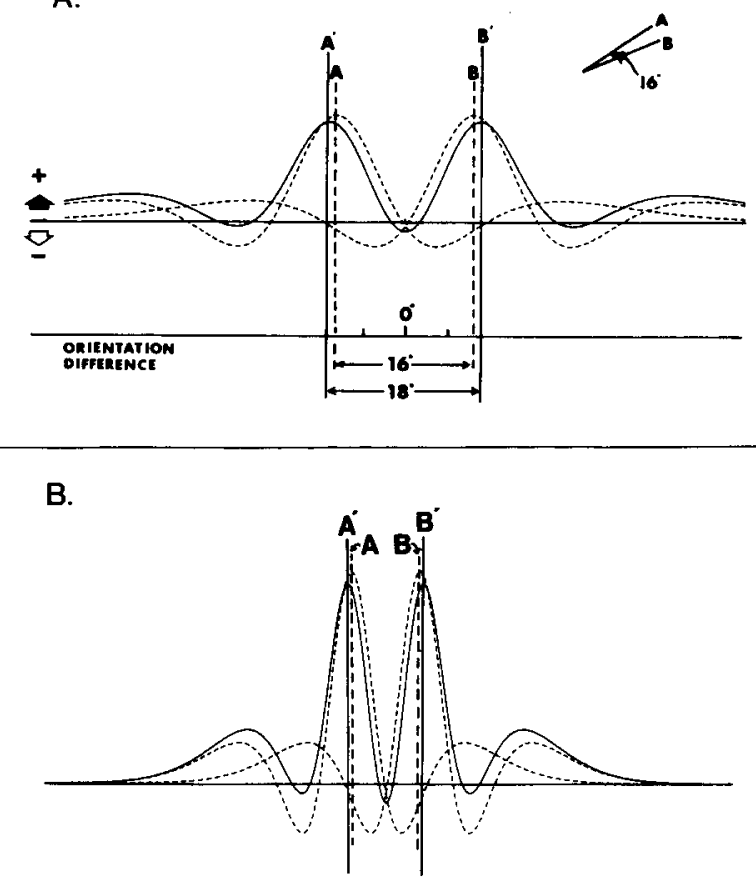

Figure 1. Hypothetical response functions (dashed lines) induced by a pair of acute-angle arms, $A$ and $B$. The distance between the two functions along the abscissa represents the difference in orientation of the two lines and the orientation tuning of the cortical cell stimulated. Positive responses are excitatory, negative responses inhibitory. Solid lines represent the linear sum of the two functions when (A) the two functions are broadly tuned and (B) narrowly tuned. Small positive sidebands are discussed later in the text. Inset shows $16^{\circ}$ angle with arms $A$ and $B$. Although the true angular separation is $16^{\circ}$, the peaks of the summed distribution (solid line) are $18^{\circ}$ apart.

increasing the luminance of $B$ increased the illusory tilt of $A$; increasing the luminance of $A$ decreased its illusory tilt; and that increasing the luminance of $A$ and $B$ jointly had little or no effect on the illusory tilt of A. In terms of Wallace's analysis, the latter result reflects the fact that increasing the luminance of both lines increases the output, and hence slope, of both excitation due to A and inhibition due to $B$, resulting in no net change in peak shift at A.

The present study concerns a stimulus parameter other than contrast or luminance which is known to affect simple cell responses in a different way but in a manner that should predictably change the shapes of the distributions in Figure 1 and so systematically change the measured tilt illusion. This stimulus parameter is line length. Unlike contrast, which increases the output of cat striate cortical cells but not their orientation selectivity (Sclar \& Freeman, 1982), increasing line length increases both the output and the selectivity of cat striate cells (Henry, Dreher, \& Bishop, 1974; Henry, Bishop, \& Dreher, 1974; Rose, 1977).

Because longer lines produce more narrowly tuned response functions in single striate cells, the population response of all cells stimulated by a longer line will also be more narrowly tuned and, hence, Figure $1 \mathrm{~A}$ represents an example of population profiles induced by relatively short lines; Figure 1B represents profiles induced by significantly longer lines.

From Figure 1, it can be deduced, first, that smaller illusions would occur with narrowly tuned functions because, when excitatory peaks are sharper, the amount by which the peaks can be shifted by inhibition is reduced; and, second, that if one were to measure the illusion as a function of acute angle size (its angular function), maximum repulsion would occur at smaller angles for more narrowly tuned response functions, because the inhibitory flank of one function would coincide with the excitatory peak of the other at smaller angular separations.

Some previous experiments (e.g., O'Toole, 1979; Wenderoth \& Johnson, 1984; Wenderoth, O'Toole, \& Curthoys, 1975) have examined the effect of joint variation of inducing and test line length on the magnitude of the tilt illusion, but typically they have used only one or two fixed angle sizes. If jointly increasing or decreasing I and $T$ line length influences both the absolute size of the illusion and the angle at which the angular function peaks, then little information can be gained by a comparison of line length effects for a single angle size. This problem of interpretation is confounded further by the fact that the position of the peak of the angular function also varies with the orientation of the acute angle relative to the main axes of space: maximum illusion occurs with smaller angles when the angle's arms are close to vertical or horizontal but with larger angles when the arms are oblique (Carpenter \& Blakemore, 1973). Consequently, very little can be inferred from the fact that O'Toole (1979) obtained larger illusions with longer but equal-length $I$ and $T$ lines when the judged arm of a $20^{\circ}$ angle was vertical, whereas Wenderoth and Johnson (1984), in another context, obtained no effect of joint variation in I and $T$ arm length when the judged arm of a $15^{\circ}$ angle was oriented $15^{\circ}$ (horizontal: $0^{\circ}$ ).

The aim of the experiments reported here was to test predictions derived from the model in Figure 1 concerning the effects of line length. Experiment 1 examined the effect of varying the length of the inducing line (I) relative to the test line $(\mathrm{T})$ and vice versa, using two angle sizes, $15^{\circ}$ and $75^{\circ}$; Experiment 2 was concerned with the effects of varying both lengths jointly and measuring the complete angular function. Although, as Parker (1974) noted, the model predicts no effect with joint variation of $I$ and $T$ luminance or contrast, this is not so in the case of line length.

\section{EXPERIMENT 1}

Robinson (1972, p. 89) reported anecdotal evidence that long $\mathrm{T}$ lines and short $\mathrm{I}$ lines result in smaller tilt illusions than short $T$ lines and long $I$ lines. The inhibition model predicts this result, as Figure 2 shows.

Figure $2 \mathrm{~A}$ represents the interaction of a short $\mathrm{T}$ line (on the left) and a long $I$ line. The illusory tilt of line $A$ (from $A$ to $A^{\prime}$ ) is relatively large, partly because the peak 
A.

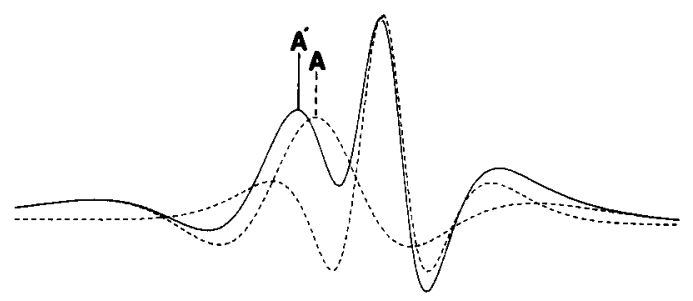

B.

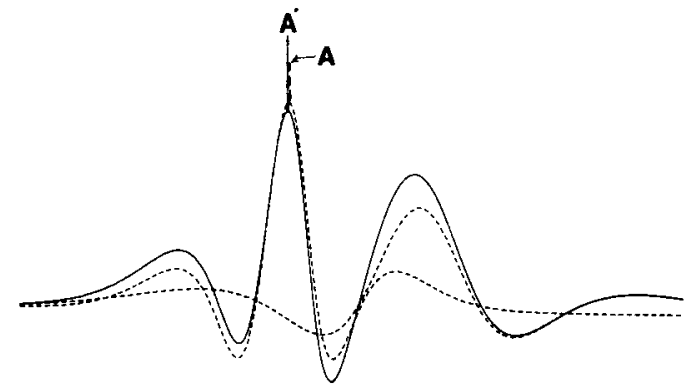

Figure 2. Hypothetical response functions induced by (A) a short $T$ line and a long $I$ line and $(B)$ a long $T$ line and a short $I$ line. The peak shift is larger in magnitude in (A) than in (B). Conventions are as for Figure 1.

of A is broad, and so there is scope for large peak shift, and partly because the long I line induces a sharp gradient of inhibition. In Figure 2B, however, the long $T$ line (again on the left) induces a sharp excitatory peak, with little scope for shift, and the short I line induces a shallow gradient of inhibition. These two factors combine to produce a much smaller illusory tilt of line A from A to $\mathrm{A}^{\prime}$.

Although O'Toole (1979) has reported already that larger tilt illusions occur with relatively longer I lines and that smaller illusions occur with relatively shorter I lines, there are reasons for our wishing to reexamine the problem. O'Toole did not use the type of display used by Robinson (1972) and by others (e.g., Carpenter \& Blakemore, 1973; Virsu \& Taskinen, 1975; Wenderoth \& Johnson, 1984, 1985), in which the I and $T$ lines abut at one end to form a single acute angle. Rather, his vertical $\mathrm{T}$ line was intersected centrally by a tilted I line.

With this kind of display, using relatively thick (unspecified) lines produced by phosphorescent powder and PVC tape, subjects may have had difficulty resolving the $\mathrm{T}$ and $\mathrm{I}$ lines, especially when both were as short as $0.5^{\circ}$.

The purpose of Experiment 1 was to examine the effect of varying the relative lengths of $I$ and $T$ lines in the abutting line tilt illusion figure. Two acute angle sizes were chosen: $15^{\circ}$ and $75^{\circ}$. The reason for including the $75^{\circ}$ angle arose from an extension of the lateral inhibition model, outlined by O'Toole and Wenderoth (1977). They suggested that if an I or T line excites cells tuned to, or tuned close to, its orientation but inhibits cells tuned to more remote orientations, then cells tuned to even more remote orientations would be disinhibited due to the inactivity of their inhibited neighbors. These disinhibitory peaks, resembling secondary excitatory peaks, are shown in Figures 1 and 2. Given sufficient angular separation of $\mathrm{I}$ and $\mathrm{T}$ lines, the disinhibitory peak of one response function would coincide with, and summate with, the nearer side of the excitatory peak of the other. This would result in the new peaks' shifting toward each other: an attraction (indirect) effect. That such effects frequently are obtained with crossing lines or inducing gratings (cf. O'Toole \& Wenderoth, 1977) but have not been reported with the abutting line display could be related to the fact that this display has usually been used with short $\left(1^{\circ}\right)$ lines (Carpenter \& Blakemore, 1973; Virsu \& Taskinen, 1975; Wenderoth \& Johnson, 1985). Longer lines, because they induce narrowly tuned response functions, might increase the chances of obtaining attraction effects because disinhibitory and excitatory peaks would summate at smaller angular separations. Therefore, $75^{\circ}$ angles were included in Experiment 1 to ascertain whether attraction or indirect effects might occur when the I line was very long. However, the main prediction was that larger illusions would occur for I $>\mathrm{T}$, smaller effects for $\mathrm{T}>\mathrm{I}$.

\section{Method}

Apparatus. Displays were produced on the flat screen of a Tektronix 608 monitor ( $\mathrm{P4}$ phosphor), interfaced with a Hewlett-Packard graphics translator (1350A) and a PDP-11/20 computer. Subjects viewed that display from a padded forehead- and chinrest centered $57 \mathrm{~cm}$ from the screen, so that $1 \mathrm{~cm}$ subtended $1^{\circ}$ of visual angle. The outer two of a set of three microswitches enabled the observer to move stimulus lines either clockwise or counterclockwise; the central switch was pressed to indicate completion and to record the response value. Pressing or tapping the switches actually moved the comparison line in $0.25^{\circ}$ increments so that continuous pressing resulted in apparent rotation of $1.9^{\circ} / \mathrm{sec}$.

Stimuli. There were 16 different angle displays, 8 for $15^{\circ}$ acute angles and 8 for $75^{\circ}$ angles. Four of the 8 stimuli, in each case, had a $2^{\circ} \mathrm{T}$ line and 4 had a $2^{\circ} \mathrm{I}$ line. The 4 stimulus figures with $2^{\circ} \mathrm{T}$ (or I) lines had $\mathrm{I}$ (or $\mathrm{T}$ ) lines $0.5^{\circ}, 1.5^{\circ}, 2.5^{\circ}$, or $3.5^{\circ}$ long. There were an additional 5 stimulus displays with the $\mathrm{T}$ line $0.5^{\circ}$, $1.5^{\circ}, 2.0^{\circ}, 2.5^{\circ}$, or $3.5^{\circ}$ long and the I line absent. These were control stimuli.

In every case, the $T$ line was oriented $45^{\circ}$, the I line joined the $\mathrm{T}$ line at its lower left end, and the center of the $0.8^{\circ} \mathrm{C}$ line, about which it rotated, was $1.2^{\circ}$ orthogonally above $\mathrm{T}$, with its lower end $0.1^{\circ}$ from the I-T vertex. The luminance of all lines, seen through a circular black mask and partially crossed Polaroid filters, was about $2 \mathrm{~cd} / \mathrm{m}^{2}$, measured with an SEI photometer. Contrast, defind as $\left(L_{\max }-L_{\min }\right) /\left(L_{\max }+L_{\min }\right)$, was close to 1.0 , and observers were dark-adapted for a few minutes prior to testing in the completely dark laboratory.

Procedure. Observers judged all 20 stimulus displays and set $\mathrm{C}$ apparently parallel to $\mathrm{T}$ four times for every display, once each from starting positions (SPs) of $\pm 5^{\circ}$ and $\pm 10^{\circ}$ from true parallelism. These 80 trials were in a different random order for each subject. The instructions encouraged subjects to bracket around the apparently parallel position before making a final setting.

Subjects. There were 15 subjects, all volunteers from an introductory course in psychology. All had emmetropic or corrected vision. 


\section{Results}

Illusions were measured as the difference between the test setting of $C$ (when the I line was present) and the appropriate pretest setting of $\mathrm{C}$ (when I was absent and the $\mathrm{T}$ line length was identical). The five pretest means did not differ as a function of T-line length: they averaged $-0.33^{\circ}$ (where the negative sign represents errors counterclockwise of zero) and had an average standard error of $0.25^{\circ}$.

Mean illusions are shown in Figure 3. The average standard error, derived from the $M S$ e in the single-factor repeated measures analysis (Winer, 1962, chap. 4) was $\pm 0.30^{\circ}$, as shown by the bar in the lower panel.

The overall result can be seen in the figure: When the I line was fixed at $2^{\circ}$ (open circles), lengthening the $T$ line decreased the illusion; when the $\mathrm{T}$ line was $2^{\circ}$ long, lengthening the I line increased the effect. This was generally true for both $15^{\circ}$ and $75^{\circ}$ angles.

Planned contrasts, all with $F(1,210)=3.94$ for $p=$ .05 , compared the pairs of means directly above or below each other in Figure 3 (e.g., $\mathrm{I}=2^{\circ}, \mathrm{T}=0.5^{\circ}$ vs. $\mathrm{I}=0.5^{\circ}, \mathrm{T}=2^{\circ}$, etc.). In the upper panel of Figure 3 $\left(15^{\circ}\right.$ angles), the two left-hand pairs were different ( $F \mathrm{~s}$
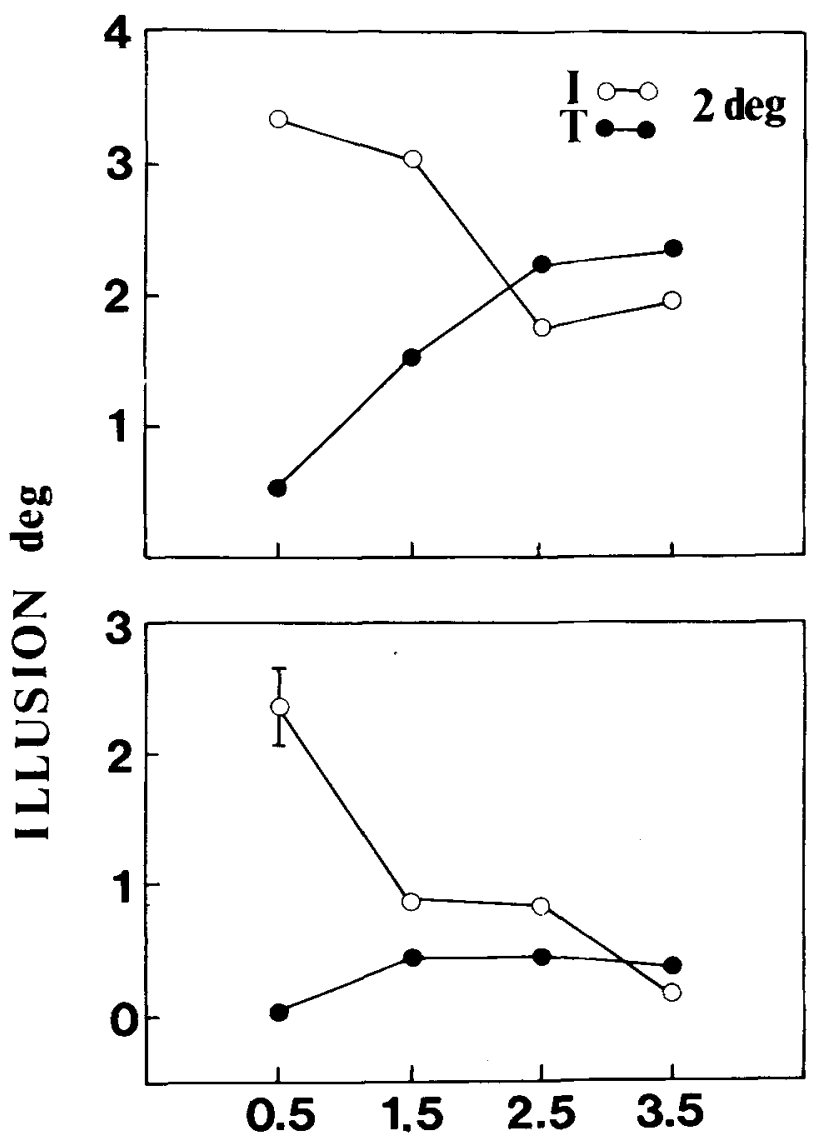

\section{I or T LENGTH deg}

Figure 3. Mean tilt illusions for varying combinations of $I-$ and T-line length, Experiment 1. Upper panel, $15^{\circ}$ angles; lower panel, $75^{\circ}$ angles.
$=42.73$ and 12.34 , respectively; $p<.0005)$, but the other two were not $(F \mathrm{~s}=1.30$ and $0.78 ; p \mathrm{~s}>.05)$. In the lower panel $\left(75^{\circ}\right.$ angles), only the left-hand pair were different $(F=29.40 ; p<.0005)$. For the other three pairs, $F \mathrm{~s}=1.05,0.74$, and $0.19, p>.05$.

One other contrast compared the overall means of the $15^{\circ}$ and $75^{\circ}$ angles, and this difference was significant $(F=84.55, p<.0005)$.

\section{Discussion}

The main finding of Exeperiment 1 was that the relative lengths of $I$ and $T$ lines affected tilt illusion magnitude as predicted by the lateral inhibition model: larger illusions occurred with longer I and shorter T lines; and smaller illusions occurred in the reverse cases. There was no indication of indirect or attraction effects with the $75^{\circ}$ angles; all illusions were positive (repulsion) effects, albeit sometimes very small.

These results are consistent with Robinson's (1972) informal observations and O'Toole's findings with small acute angles.

\section{EXPERIMENT 2}

O'Toole (1979) based all of his predictions on the fact that longer $I$ and $T$ lines would have a larger inhibitory output and did not consider the relevance to the model of the effect of line length on orientation selectivity. Although the results of Experiment 1 were consistent with $O$ 'Toole's data for I and T lines of unequal length, it is of interest to note that when, in $O$ 'Toole's experiment, I and $\mathrm{T}$ lines were equal in length $\left(0.5^{\circ}, 1.0^{\circ}\right.$, or $2.0^{\circ}$ long), he found that the tilt illusion increased with the joint increase in the length of $\mathrm{I}$ and $\mathrm{T}$ (effects of $0.26^{\circ}, 0.66^{\circ}$, and $0.94^{\circ}$, respectively).

This result is puzzling: If line length changed only the output of cells and not their selectivity, then there should have been little or no effect of jointly increasing I and T length, as Parker (1974) predicted and found using luminance as the parameter. When both output and selectivity are considered, that model seems to predict the opposite of O'Toole's result, namely, a decrease in the illusion as joint length increases. This can be seen by comparing the illusory effects (A to $A^{\prime}$ ) for short lines (Figure 1A) and long lines (Figure 1B). Another prediction can be generated from Figure 1: Since the maximum tilt illusion should occur when the inhibitory trough due to the I line coincides with the excitatory peak due to the $T$ line, the angular separation between $I$ and $T$ should be less for maximum illusion when the lines are long than when they are short, because longer lines narrow the population tuning functions.

Experiment 2 aimed to test these predictions by measuring the angular functions of tilt illusions when both I and $\mathrm{T}$ lines were $1^{\circ}, 2^{\circ}$, or $3^{\circ}$ in length.

\section{Method}

Apparatus, stimuli, and procedures essentially were the same as in Experiment 1, except that all angular figures had equal-length arms. The comparison line always was positioned near the vertex, 
as in Experiment 1, regardless of the lengths of $I$ and $T$. All subjects judged all three angle-arm sizes and all angular separations of I and T, providing two judgments of each display from SPs of $\pm 10^{\circ}$.

The experiment actually was run twice, for reasons that will be discussed, once using 10 angle sizes from $0^{\circ}$ (pretest control) to $90^{\circ}$ in $10^{\circ}$ steps and once using 10 angle sizes of $0^{\circ}, 10^{\circ}, 15^{\circ}$, $20^{\circ}, 25^{\circ}, 30^{\circ}, 75^{\circ}, 80^{\circ}, 85^{\circ}$, and $90^{\circ}$.

The first run of the experiment used 15 volunteers drawn from the same population as those in Experiment 1 ; the second run used 10 subjects, 9 from an advanced undergraduate course who completed the experiment as an assignment but were naive regarding the experimental aims, and the first author.

\section{Results}

The first run of the experiment produced the results shown in Figure 4, in which the angular functions shown are test minus pretest values. The actual pretests obtained are shown by the single symbols at $0^{\circ}$ on the abscissa. Although the results seemed at first consistent with the predictions that longer lines would induce both smaller illusions and a peak at smaller angles, analysis of the data gave some cause for concern. First, the pretest mean for $3^{\circ}$ lines $\left(+0.55^{\circ}\right)$ was significantly different from that for $1^{\circ}$ lines $\left(-0.50^{\circ}\right)[F(1,406)=8.143, p<.005]$. Consequently, the apparent difference in magnitude between the $1^{\circ}$ line length effects (solid circles) and the $3^{\circ}$ line length illusions (solid squares) could have been due entirely to this pretest difference. When the peak illusions for $1^{\circ}$ and $3^{\circ}$ lines were compared in the uncorrected data (i.e., pretest not subtracted), there was no significant difference between the largest $1^{\circ}$ line ef- fect $\left(2.38^{\circ}\right)$ and the largest $3^{\circ}$ line effect $\left(1.92^{\circ}\right)[F(1,261)$ $=0.83, p>.05]$. A second problem was that although the $1^{\circ}$ line function peaked at $20^{\circ}$, both the $2^{\circ}$ and $3^{\circ}$ line functions peaked at $10^{\circ}$ : the steps of the angle sizes chosen were too coarse to discriminate the predicted ordering.

To test statistically for differences in the positions of the peak effects, the angles at which each subject's two largest illusions occurred were averaged. For example, if a subject's largest effect occurred at $10^{\circ}$ and the second largest effect at $20^{\circ}$, the peak was recorded as $15^{\circ}$. The result of this analysis was that the mean peaks for the $1^{\circ}, 2^{\circ}$, and $3^{\circ}$ line functions were $24.67^{\circ}, 23.00^{\circ}$, and $23.33^{\circ}$, with standard errors of $2.4,1.8$, and 1.9 , respectively. A contrasts analysis showed that the $1^{\circ}$ and $3^{\circ}$ peaks were not different $[F(1,28)=0.32, p>.05]$ and that the $2^{\circ}$ peak was not different from the mean of the other two $(F=0.24, p>.05)$.

The results of the second run of the experiment, in which smaller step sizes in angle were chosen to increase discrimination between peak positions, are shown in Figure 5. This time, there was no significant difference between the $1^{\circ}$ and $3^{\circ}$ pretests $[F(1,261)=0.04$, $p>.05$ ] and the $2^{\circ}$ pretest did not differ from the means of the other two $(F=0.29, p>.05)$.

The largest mean illusions for the $1^{\circ}, 2^{\circ}$, and $3^{\circ}$ functions were $2.01^{\circ}, 2.13^{\circ}$, and $2.21^{\circ}$, respectively. The first and last of these were not different $[F(1,261)=0.11$, $p>.05]$, nor was the average of these two different from the other mean $(F=0.003, p>.05)$.

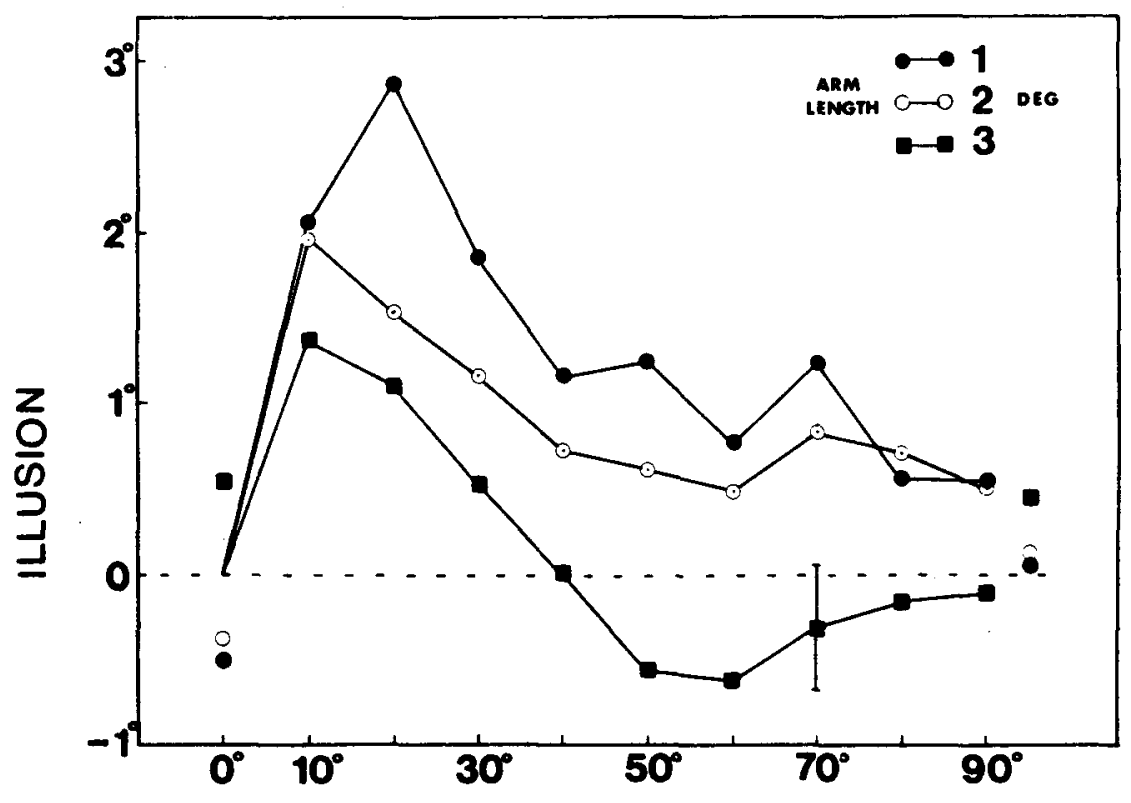

\section{INDUCING ANGLE}

Figure 4. Angular functions of tilt illusions as a function of $I$, $T$ line length, Experiment 2, first run. Error bar shows $\pm 1 \mathrm{SE}$. Functions are for $1^{\circ}$ lines (solid circles), $2^{\circ}$ lines (open circles), and $3^{\circ}$ lines (solid squares). 


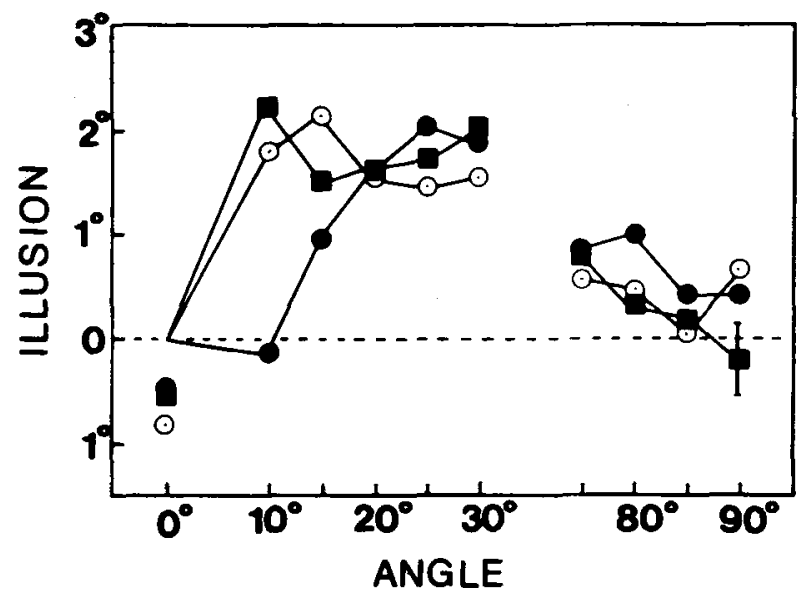

Figure 5. Angular function of tilt illusions as a function of $I, T$ length, Experiment 2, second run. Symbols are as for Figure 4. Error bar shows $\pm 1 \mathrm{SE}$.

The analysis for peak position, similar to that carried out on the first-run data, yielded peak position estimates for the $1^{\circ}, 2^{\circ}$, and $3^{\circ}$ functions of $24.75^{\circ}, 20.05^{\circ}$, and $19.75^{\circ}$ with standard errors of $0.69,1.17$, and 1.60 , respectively. The first and last of these were significantly different $[F(1,18)=12.53, p<.005]$. The average of these two means was not different from the other mean $(F=3.23, p>.05)$.

The slightly negative illusion at $10^{\circ}$ for the $1^{\circ}$ figure was not expected. However, 6 of the 10 subjects obtained positive illusions in that condition, and 2 of those whose illusions were negative had effects of $-2.5^{\circ}$ and $-3.0^{\circ}$, which greatly reduced the overall mean.

Another feature of the results that was surprising was the apparent tendency of the $3^{\circ}$ arm illusions to rise to an apparent second peak at $30^{\circ}$. Further research is required to establish whether this bimodality is real and, if so, how it might be explained.

Finally, there was no evidence of assimilation (negative) effects for the larger acute angles. It is perhaps worth noting that the differences between the two runs of Experiment 2 essentially are attributable to the differences in the pretest means between the two experiments: subtraction of the first-run pretests from the second-run test data produces the same ordering of peaks, ordering of magnitudes, and even the apparent long-line indirect effects shown in Figure 4.

\section{GENERAL DISCUSSION}

The experiments reported here are consistent with some of the predictions of the lateral inhibition model of the tilt illusion. Based upon physiological evidence, it was suggested that short lines would produce a broad response function in cortical orientation-selective neurons whereas long lines would produce a more narrowly tuned popula- tion response. This, in turn, was taken to imply that small tilt illusions would occur when the inducing line was short relative to the test line, and that larger illusions would occur when the test line was short relative to the inducing line. The results of Experiment 1 were consistent with this prediction.

A second prediction was that when both inducing and test lines were short, the angular function of the tilt illusion would peak at a larger angular separation of $I$ and $T$ lines than when both lines were long. The analysis of the second run of Experiment 2 generally was consistent with this prediction.

The third main prediction was not borne out by the experiments. The model predicted that angles with both arms long would induce smaller effects than angles with both arms short. Although the first run of Experiment 2 seemed to confirm this prediction, differences between the pretest (control) means for the three line length conditions accounted virtually entirely for the differences in illusion magnitude. In the second run, in which no such ordering of pretest mean magnitudes occurred, no significant differences were obtained in illusion magnitudes as a function of line length.

In some ways, the failure to confirm this latter prediction is not surprising. The notion that the perceived orientation of a line depends simply upon the orientation tuning of the most active neurons stimulated by it has always raised problems. In formulating their model, Carpenter and Blakemore (1973) had to allow for the fact that illusions can be as large as $3^{\circ}$; but they had also to allow for the fact that illusions occur at quite small angular separations. As a result, their hypothetical population response profiles were required to appear, as they themselves noted, rather unphysiological, with broad flat peaks (at least $6^{\circ}$ wide to allow $\pm 3^{\circ}$ peak shifts) and very abrupt transitions (slopes) from excitation to inhibition (to allow for small-angle illusions). It might be useful to explore alternatives to the simple "peak shift" model, perhaps taking into account the mean or median of the response functions and even considering their skew.

One other prediction tested in these experiments was that based on the extended model of O'Toole and Wenderoth (1977), namely, that longer I and $T$ lines might be more likely to induce attraction or indirect illusions with larger acute angles. This is not only predicted by the extended model but makes some intuitive sense: any stimulus parameter, such as line length, which causes the illusion's angular function to peak earlier (at smaller angles) should result in the illusion's decreasing to zero earlier, so providing more opportunity for negative crossings. Although a semblance of such negative effects occurred in the initial run of Experiment 2, they were due, again, to the positive pretest, and no such effects were found in the second run. However, perhaps further investigation, with the $\mathrm{T}$ line at orientations other than $45^{\circ}$, is warranted. 


\section{REFERENCES}

Carpenter, R. H. S., \& Blakemore, C. (1973). Interactions between orientations in human vision. Experimental Brain Research, 18, 287-303.

Henry, G. H., Bishop, P. O., \& Dreher, B. (1974). Orientation, axis and direction as stimulus parameters for striate cells. Vision Research, 14, 767-777.

Henry, G. H., Dreher, B., \& Bishop, P. O. (1974). Orientation specificity of cells in cat striate cortex. Journal of Neurophysiology, 37, 1394-1409.

O'TOOLE, B. I. (1979). The tilt illusion: Length and luminance changes of induction line and third (disinhibiting) line. Perception \& Psychophysics, $25,487-496$.

O'TOOLE, B. I. , \& WeNDEROTH, P. (1977). The tilt illusion: Repulsion and attraction effects in the oblique meridian. Vision Research, 17, 367-374.

Parker, D. M. (1974). Evidence for the inhibition hypothesis in expanded angle illusion. Nature, 250, 265-266.

RoBinson, J. O. (1972). The psychology of visual illusion. London: Hutchinson.

Rose, D. (1977). Responses of single units in cat visual cortex to moving bars of light as a function of bar length. Journal of Physiology, 271, 1-23.
Sclar, G., \& Freeman, R. D. (1982). Orientation selectivity in the cat's striate cortex is invariant with stimulus contrast. Experimental Brain Research, 46, 457-461.

VIRSU, V., \& TASKINEN, H. (1975). Central inhibitory interactions in human vision. Experimental Brain Research, 23, 65-74.

Wallace, G. K. (1975). The effect of contrast on the Zöllner illusion. Vision Research, 15, 963-966.

Wenderoth, P., \& Johnson, M. (1984). The effects of angle-arm length on judgments of angle magnitude and orientation contrast. Perception \& Psychophysics, 36, 538-544.

WENDEROTH, P., \& JohnSON, M. (1985). What is the appropriate control for the tilt illusion? Perception, 14, 275-283.

Wenderoth, P., O'ToOle, B., \& Curthoys, I. (1975). The effect of inducing line length on the magnitude of the tilt illusion and aftereffect. Australian Journal of Psychology, 26, 1-7.

WINER, B. J. (1962). Statistical principles in experimental design. New York: McGraw-Hill.

(Manuscript received October 21, 1985; revision accepted for publication April 17, 1986.) 